\title{
Advances in the Research of Yunnan's Arid Climate and Extreme Drought
}

\author{
Juzhang Ren ${ }^{1,2}$, Wancheng Zhang2*, Yunxia Wan ${ }^{3}$, Yan Chen ${ }^{2}$ \\ ${ }^{1}$ Institute of Arid Meteorology, CMA, Lanzhou, China \\ ${ }^{2}$ Meteorological Sciences Institute of Yunnan Province, Kunming, China \\ ${ }^{3}$ Yunnan Speciality Meteorological Observatory, Kunming, China \\ Email: renjzhyn@163.com, ${ }^{\star}$ hhzth@sina.com
}

How to cite this paper: Ren, J.Z., Zhang, W.C., Wan, Y.X. and Chen, Y. (2017) Advances in the Research of Yunnan's Arid Climate and Extreme Drought. Atmospheric and Climate Sciences, 7, 23-35. http://dx.doi.org/10.4236/acs.2017.71003

Received: November 17, 2016

Accepted: January 8, 2017

Published: January 11, 2017

Copyright (C) 2017 by authors and Scientific Research Publishing Inc. This work is licensed under the Creative Commons Attribution International License (CC BY 4.0). http://creativecommons.org/licenses/by/4.0/

c) (i) Open Access

\begin{abstract}
With the global climate change, the extreme drought was increasing. From 2009 autumn to 2010 spring, a hundred-year drought happened in Yunnan province, which caused great local economic losses and widespread attention. So many researches about Yunnan drought were studied. The climatic characteristics of the drought over Yunnan are studied by analyzing the spatial and temporal distribution of some meteorological factors such as precipitation, temperature and sunlight, etc. Some researchers studied the formation mechanism of the drought events in Yunnan. In this paper, by investigating lots of related documents, we had a summarization and commentary about the recent study achievements of Yunnan drought and tried to offer reference to the study on the Yunnan drought in the future.
\end{abstract}

\section{Keywords}

Yunnan, Arid Climate, Extreme Drought, The Atmospheric Circulation, Change Characteristic

\section{Introduction}

Yunnan is situated in the low-latitude plateau with complex geography and climate. Yunnan has very distinctive dry and wet seasons. It is generally thought that the rainy season is May-October, and dry season ranges from November to the next April (up to half year). According to the climatic data statistics, in the Yunnan region, the precipitation in the dry season accounts for $16 \%$ of the precipitation in the whole year [1], and the average daily precipitation during this period (average level throughout Yunnan Province) is only $0.93 \mathrm{~mm}$ (which is far lower than the evaporation in the same period). Therefore, drought is frequent in Yunnan, and there is the saying that "there is drought in nine of ten years". Yunnan is the province with the severest drought in southwest China region and is also one of the regions with frequent drought in China [2]. Under 
the context of global warming, the extreme weather and climatic hazard events have been constantly aggravated, and the extreme drought events once in a hundred years or in dozens of years happen frequently. Compared with other natural hazards, drought is characterized by high frequency, long duration and wide scope of influence, thus having huge impact on the national economy, particularly the agricultural production. In 2009/2010, super-severe drought happened in Yunnan and Guizhou, and the duration of drought in Yunnan indicated the super-severe drought in the local place since there was the meteorological record. This extreme drought event had wide scope of influence, and till May 31, 2010, 25.12 million persons in Yunnan Province suffered hazards, in which 7.57 million persons had the difficulty in obtaining the drinking water; the drought also caused the agricultural crops of 2.174 million Hectare to be affected in the autumn and winter, and direct economic loss over RMB 20 billion. This has aroused high emphasis of the government and meteorological scholars, and the related research work has been deeply carried out. The authors combed these research results, to provide the reference for subsequent study of drought.

\section{Climatic Change Characteristics in Yunnan}

The disaster-resulting factor of drought hazard is the meteorological factor, which mainly depends on the precipitation, atmospheric temperature and evaporation in a region. But with the aggravation of globalization and climatic warming, the meteorological change response in different regions was different.

\subsection{The Change Characteristic of Temperature in Yunnan}

In recent one hundred years, the average temperature-time trend has shown that, except the decrease of $-0.092^{\circ} \mathrm{C} / 10 \mathrm{a}$ in Dali City, other regions is the increasing trend, Puer City in the south of Yunnan Province has the largest growth amplitude, that is, $0.105^{\circ} \mathrm{C} / 10 \mathrm{a}$, which is followed by Shangri-La in the northwest of Yunnan, that is, $0.079^{\circ} \mathrm{C} / 10 \mathrm{a}$. Before the $1980 \mathrm{~s}$, Puer City and Shangri-La were in the relatively cold period, and since the 1990s, the temperature has grown rapidly [3].

From the average growth amplitude of atmospheric temperature in Yunnan Province from 1961-2007, as show in Figure 1, it may be seen that in middle Yunnan, south Yunnan, southwest Yunnan, northwest Yunnan and southwest of west Yunnan, the temperature rises above $1.18^{\circ} \mathrm{C}$, and up to $2.08^{\circ} \mathrm{C}$ in Kunming. In addition, the growth amplitude is obviously higher than the average national level of $1.1^{\circ} \mathrm{C}$ calculated by Ding Yihui et al. [4]. In Dali and Lijiang of middle Yunnan and west Yunnan, the temperature rises slowly and is $0.4^{\circ} \mathrm{C}-0.91^{\circ} \mathrm{C}$, which is lower than the average national level. In addition, in some regions of east Yunnan, northeast Yunnan, south Yunnan, middle Yunnan and west Yunnan, the annual reduction amplitude happened with 39.3 $198.1 \mathrm{~mm}$, mostly in northeast Yunnan; while in some regions of northwest Yunnan, southeast Yunnan and southwest Yunnan, the precipitation growth amplitude was 16.7 $133.8 \mathrm{~mm}$, and the regions with the largest growth amplitude happened in Tengchong in southwest of west Yunnan and northwest Yunnan region. Due to complex topography in Yunnan Province, it is largely influenced by the monsoon, there are quite different climatic regions, and the atmospheric temperature and precipitation in the regions 


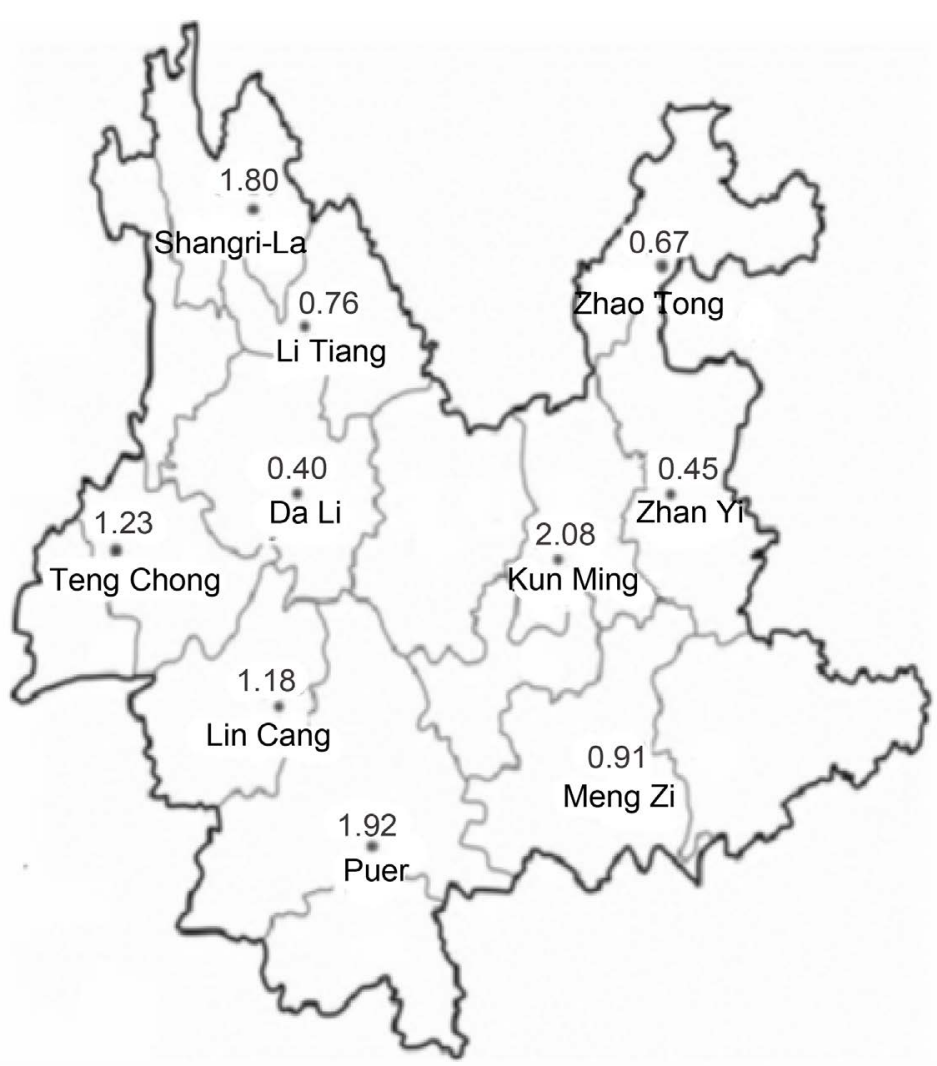

Figure 1. (Select from literature 4). The amplitude of annual average temperature for 47 years in Yunnan.

have different change trends in different seasons.

The temperature warming trend is related to the diurnal range of atmospheric temperature in different climatic belts in Yunnan. The growth rate of minimum atmospheric temperature bigger than that of maximum atmospheric temperature in north Asian tropic, central Asian tropic and south Asian tropic stations, so the diurnal range of temperature obviously decreases. But the change of the diurnal range of temperature in the temperate zone and north tropic zone isn't significant [5]. The distribution of maximum atmospheric temperature in Yunnan region generally increases with the decrease of the latitude from northwest Yunnan to south Yunnan, with the change amplitude in $11.5^{\circ} \mathrm{C}-30.7^{\circ} \mathrm{C}$. And it also manifests obvious regional difference, such as in the south of Yunnan, dry-hot valley basin (mainly including Jinsha River, Lancang River, Yuanjiang River and Nujiang River etc.), and center of Yunnan, there is the high temperature center, and the maximum temperature center is situated in Yuanjiang River in the Yuanjiang River Basin valley region, with the temperature of $30.6^{\circ} \mathrm{C}$. Except the complex landform, that is, the "channel-obstruction" effect of the landform [6], the space distribution of the maximum atmospheric temperature is influenced by the altitude of the observation station [7]. In Yunnan Province, the average change trend of annual maximum atmospheric temperature has the obvious rise trend, with the rise rate of $0.17^{\circ} \mathrm{C} / 10 \mathrm{a}$. In the inter-annual change, the extremely low value of the maximum atmospheric temperature $\left(22.3^{\circ} \mathrm{C}\right)$ happened in 1976 , but the extremely high value $\left(24.2^{\circ} \mathrm{C}\right)$ happened in 2010 , and the super-severe drought once in hundred years 
happened in the same year.

\subsection{The Change Characteristic of Precipitation in Yunnan}

The objective analysis of fine grate is made with the atmospheric temperature and precipitation information in 124 stations in Yunnan Province in 1961-2008 (the temperature interpolation considers the topographic height correction), and on this basis, the atmospheric temperature and precipitation sequence has received the space correction and climatic zoning [8]. In recent 50 years, the atmospheric temperature rose most in the winter in Yunnan, then in autumn, summer and spring, but there is the falling trend in some low-altitude valley area. Average annual change of precipitation isn't large, the precipitation in the rainy season decreases, and the precipitation in the dry season rises. Liu Yu et al. [9] drew the conclusion that the precipitation in the summer in 1961-2007 obviously decreased by analysis with the linear trend method, with the decrease rate of $4.5 \mathrm{~mm} / 10 \mathrm{a}$, and the sudden change of the precipitation from rise to fall had been happened about in 1971; the growth rate of atmospheric temperature in the winter was $0.27^{\circ} \mathrm{C} / 10 \mathrm{a}$, and there was a sudden change from cold to warm in 1995 . Regarding the regional distribution characteristics of precipitation throughout Yunnan Province, the uniform decrease happened in the summer, the precipitation decreased in the southwest Yunnan and increased in other regions in the winter, the uniform increase happened in the spring, and the precipitation in the west increased and decreased in the east in the autumn [10].

\subsection{Change Characteristic of Evaporation Capacity in Yunnan}

Solar radiation is the main factor influencing the change of evaporation [11], the sunshine hours can express the main constitution of solar radiation energy in one position to certain extent [12] [13] (that is, direct solar radiation quantity), is the energy source for the ground surface, water surface and air, and is often in positive correlation with the average temperature and ground temperature, as show in Figure 2. In Yunnan Province, the distribution of sunshine hours is generally characterized by much in west Yunnan, little in east Yunnan and more in the south than that in the north, and the maximum center mainly happens in the valley zone of Jinsha River Basin. The difference of the maximum and minimum values of the sunshine hours in Yunnan is about $1600 \mathrm{~h}$. Since 1961, the change of annual sunshine hours in Yunnan is characterized by decrease in the north and increase in the south, basically taking Ailao Mountain as the boundary, with the growth trend in the west and south of Ailao Mountain, and decrease trend in the north and east of Ailao Mountain [14].

\section{Change Characteristics of Aridclimate in Yunnan}

Drought is the phenomenon of water deficiency due to unbalance of water moisturebudget or supply-demand. In the natural disasters in China, drought is always one of the severest disasters. At present, there are many indexes for the analysis and study of drought, and new indexes come out one after another. The causes are mainly closely related to the regional difference of the weather and climates and the complexity of drought problems. Meteorological drought indexes mainly include precipitation anomaly 


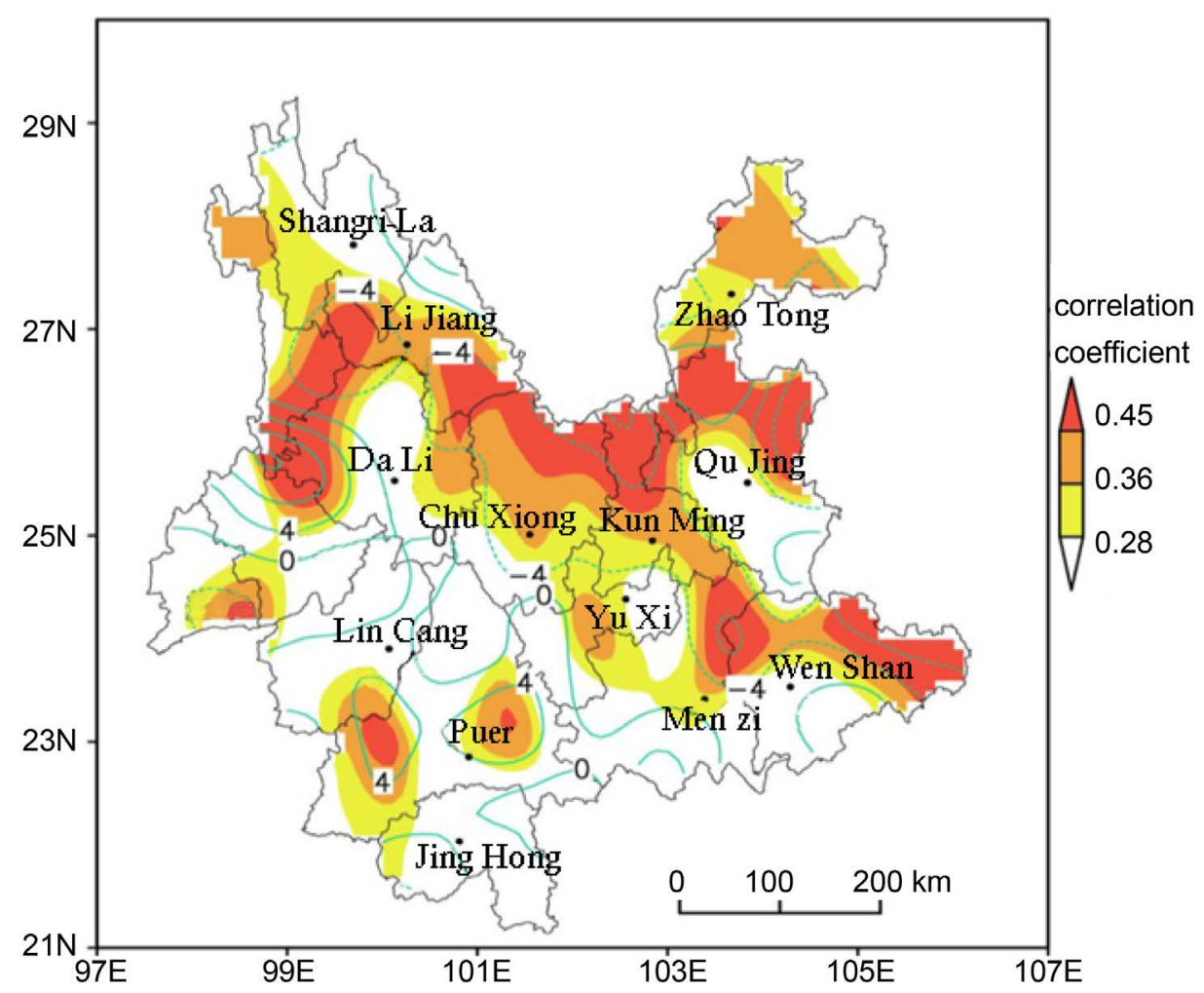

Figure 2. (Select from literature 14). Yunnan change trend of annual sunshine duration distribution and correlation coefficient.

percentage, relative moisture degree index, standard precipitation index, soil relative humidity index, and Palmer drought severity index, as well as the comprehensive meteorological drought index. Huang Zhongyan et al. [15] compared the applicability of meteorological drought index, relative moisture degree index, comprehensive meteorological drought index, precipitation anomaly percentage and standard precipitation index in the agricultural drought monitoring and assessment, thinking that the capacity of meteorological drought index and relative moisture degree index in distinguishing the drought and wetness is superior to the other three indexes. According to the indicative meaning of this index, Ren Juzhang et al. [16] use the relative moisture degree index in 1961-2010 to analyze the space distribution of drought and wetness in the dry/rainy season in Yunnan Region. The drought in Yunnan in the rainy season has the periodical change of 10 - 16 years, 6 - 8 years and 2 - 4 years, and the drought in Yunnan in the dry season mainly has the periodical change of 8 years and $4-6$ years. Since the start of 21st century, the rainy season in Yunnan gradually becomes dry, and the drought in the dry season slightly increase. This characteristic change mainly results from the non-uniform distribution of precipitation and climate warming in Yunnan since the 1990s [17], and continuously abnormal drought has happened in Yunnan since 2000 year. The single-station comprehensive drought index [18] is used to assess the drought disaster in Yunnan. The drought in January-March is the severest, and about $2 / 3$ of the land suffers the drought every year; it is followed by November-December, in which about $50 \%$ of the land suffers drought, and in first ten days of April June, $22 \%$ of the land suffers drought; in September-October, only $5 \%$ of the land suf- 
fers drought, and in June-August, less than $1 \%$ of the land suffers drought. From analysis on the drought area, it is known that in first ten days of April-June and September-October, drought has the trend of climatic change for development and aggravation.

The change characteristics of multiple drought indexes have indicated that the drought in Yunnan has the trend of climatic change for development and aggravation, and the drying in Yunnan in the rainy season becomes more obvious. In such climatic context, it is easy to have continuous seasonal drought in Yunnan, just as the continuous drought in the autumn, winter, spring and early summer of 2009-2010, and the relative moisture degree index was obviously smaller in the rainy season of 2009 (or in the later period of the rainy season) (and there was no drought in the rainy season), which is followed by obviously low moisture degree index in the dry season (reflected by typical drought in the winter and spring), and in addition, the rainy season in 2010 started late (reflected by drought in the early summer), resulting in the strongest drought intensity in 2009-2010 since there is the meteorological record and also resulting in the largest disaster and economic loss. After comparing the other three extreme droughts in history (1962/1963, 1968/1969 and 1978/1979), all were continuous seasonal drought, mainly happening in the central and east regions of Yunnan, characterized by long duration and uniform distribution [19].

\section{Causes of Drought in the Low-Latitude Plateau}

\subsection{Causes of Drought in the Early Summer}

May is the transfer period of the dry and wet seasons, and the precipitation amount and the start time of the rainy season are closely related to the agricultural production. Generally speaking, if the rainy season starts earlier with more precipitation, it is more beneficial for the planting of crops sown in the spring, and severe drought in May will influence the output and quality of crops sown in the spring. Therefore, the precipitation in May and the forecast of the start of the rainy season are critical for the meteorological department to provide the decision service for the governments in the levels, and is critical for the short-term climatic forecast in Yunnan.

The rainy season in Yunnan generally happens in the fifth pentad of May. If it is advanced or postponed with two pentads, it is called as the early or late start of the rainy season, and if it is advanced or postponed with three pentads, it is called as quite early start or quite late start. The main causes resulting in early or late start of the rainy season are obvious in the abnormal characteristics of atmospheric circulation in May. In case of the late rainy season, the Eurasian medium-high latitude has two troughs and one ridge in $500 \mathrm{hPa}$ height field in May, and the seasonal northward movement of the westerly is late. The cold air influencing Yunnan is weak, the subtropical anticyclone of West Pacific in the low level is westward, the northward movement of the equatorial westerly is obstructed, resulting in late outbreak and relatively weak intensity of the summer monsoon in the Bay of Bengal and Indochina Peninsula. Yunnan is situated in the sinking branch of Hadley circulation, where the vapor flux is characterized by abnormal divergence, and the water vapor content is smaller than that in the previous years. In case of early start of the rainy season, the circulation characteristics in May are 
on the contrary [20].

Such abnormal atmospheric circulation has resulted in continuous little rain and high temperature in May, 2005, forming relatively severe drought. In May, 2005, the whole season transfer is late, the average position of subtropical westerly jet is 6 - 7 dimensions southward by comparison with the same period in history, and the season transfer is obviously postponed, till the South Asia high is stably built on June 5, and the start time of the rainy season is obviously postponed [21].

The influence of such abnormal circulation is closely related to the abnormal sea temperature of the Pacific and Indian Ocean in the previous period and in the same period [22] [23]. In the current period, the fall (rise) of the sea temperature in the South Indian Ocean is beneficial (unbeneficial) for the intensification of convection and the formation of the low-pressure system of Bengal region in the early summer, and the low pressure in the Bay of Bengal happens frequently in the early summer, thus the start time of the rainy season in Yunnan is earlier [24]. After the comparison and analysis of the circulation characteristics in May in the drought year and rainy year, it is mainly featured by the westerly vapor transportation in Yunnan in the drought year, which corresponds to the abnormal vapor flux divergence, and the vapor content is less than that in the previous year, and the transfer of the dry and wet seasons is late and is heavily influenced by the inter-annual change of the monsoon intensity in Asia, in which the South Asia high differs obviously and the South Asia high is obviously weak in the drought year [25]. ENSO event on the Pacific Ocean has obvious influence on the start time of the rainy season in Yunnan, and in the EL Nino year, the start time of the rainy season in Yunnan is late, and among 14 EL Nino events, the start time of the rainy season in Yunnan for eight times happened in the sixth pentad of May and later. While in the La Nina year, it corresponds to the early start of the rainy season, and in ten years, there are seven years with normal early start [26].

\subsection{Causes of Drought in the Autumn}

Autumn is the transitional season from rainy season to dry season in Yunnan, and precipitation is more closely coupled with vapor, and has the distribution characteristics of vector field related to "cyclone-anticyclone" with the whole layer of vapor flux [27]. In the above space of the southwest region, the distribution of the precipitation is differently related to the precipitation in Yunnan in the dry and rainy seasons, and the precipitation in Yunnan in the autumn is in positive correlations with the vapor content in the above space [28] [29]. Therefore, the discussion on the drought in the autumn mostly focuses on the impact of abnormal vapor on the autumn in Yunnan.

With respect to the vapor budget, the precipitation in the autumn in Yunnan is mostly influenced by the vapor inflow in the west and south boundary. In September, the vapor inflow in the west and south boundary in the positive abnormal precipitation years is more than that in the negative abnormal precipitation years, and the outflow in the north boundary is less. In October, the vapor inflow in the positive abnormal precipitation years has the largest difference in the south boundary by comparison with that in the negative abnormal precipitation years. In November, the large difference of vapor flux in the positive and negative abnormal precipitation years happens in the upper 
layer of the north and east boundary and the whole layer of the west and south boundary [30]. The vapor in the west and south boundary mainly comes from the Indian Ocean and Bay of Bengal, and is transported northward to the southwest region by depending on the monsoon circulation system, thus influencing the precipitation in the autumn in Yunnan, and the formation of drought. If the southwest monsoon and south trough in the autumn are active, the southerly warm and wet air flow influencing Yunnan will become strong and there will be more precipitation in the autumn of Yunnan, otherwise the precipitation will become less, which easily results in drought.

In the autumn of 2009, the height field and circulation between the Bay of Bengal and South China Sea had the asymmetrical change, so that in the space above these two places, the air pressure gradient is abnormally reduced, which has resulted in the abnormal decrease of southwest airflow between the low pressure of the Bay of Bengal and the high pressure of the South China Sea, and the vapor in the southwest region of China also decreases, and the region between the southwest of China and the Central South Peninsula is under the control of northerly anomaly wind, in the space above the southwest region, there is the abnormal sinking movement, thus the convection movement is inhibited, resulting in the drought in the autumn in southwest China region [31].

\subsection{Causes of Extreme Drought}

In recent years, severe drought events happened in Yunnan and its neighboring zones continuously, particularly the drought in the autumn, winter and summer of 2009/2010 and 2011/2012 aroused strong repercussion, and many scholars carried out the work of studying the causes of extreme drought. Sun Guowu et al. [33] compared and analyzed the atmospheric low-frequency change characteristics in these two extreme drought years and one non-drought year. Results hold that, there are two flow types for the atmospheric low-frequency change in Yunnan region in the winter and spring: latitude in allow-frequency air flow and longitudinal low-frequency air flow. The former obstructs the north-south exchange of north (south) cold (warm) air flow; the latter ensures that there is no air flow convergence in the single north-south cold and warm air mass. Besides, in the drought year, the low-frequency anticyclone is far more than low-frequency cyclone in southwest region and India-Bay of Bengal region, the northerly air flow in front of the low-frequency anticyclone prevents the northward transportation of the vapor in the Bay of Bengal; in the non-drought year, the low-frequency air flow and the low-frequency system are opposite. Therefore, the change of atmospheric low-frequency air flow and low-frequency system might be one of the reasons resulting in the continuous drought in the winter and spring in Yunnan.

Yunnan is situated in the low-latitude region, and is sensitive in the response to the tropical abnormal circulation. The continuous abnormality of oscillation in the tropical atmospheric season is also one of the reasons for continuous drought in Yunnan. In 2009-2010, one of the main reasons for continuous drought in the autumn, winter and early spring is continuous abnormality in the season in MJO (Madden-Julian Oscillation) [34]. In June-October, 2009, the tropical Middle East and Indian Ocean MJO (Madden-Julian Oscillation) index manifests continuous positive abnormality, and cor- 
respondingly, atmospheric circulation is characterized by being continuous weak of the convection movement in the Bay of Bengal, and abnormal sinking air flow is triggered in the tropical Indian Ocean region, so that the vertical circulation cell of the Asian monsoon is abnormally weakened in South Asian region, and the vapor transported from the tropical Indian Ocean to Yunnan is abnormally reduced, resulting in continuous decrease of the precipitation in Yunnan from the summer to the autumn in 2009, so drought is formed. In 2009, the winter tropical Middle East and Indian Ocean MJO index maintained positive abnormal status, and the corresponding atmospheric circulation abnormality is maintained, that is, the convection movement in the Bay of Bengal is still weak, the abnormal high pressure ridge appeared from South Asia via Qinghai-Tibet Plateau to the southwest region, Yunnan is situated under the control of vertical sinking air flow of high pressure ridge, which is unbeneficial for the generation of convection movement. Besides, the south trough in the winter has weak intensity, which is unbeneficial for the movement from the Bay of Bengal to Yunnan. So in Yunnan, the precipitation is small in the winter, and drought is intensified, thus forming continuous drought in the autumn, winter, spring and early summer.

After the diagnosis of atmospheric circulation difference in the extreme event years with continuous seasonal drought, it is found that in the seasonal continuous drought year, the abnormality of the high-latitude Arctic Oscillation (AO) also has influence on the drought in Yunnan. The correlation of $\mathrm{AO}$ with the precipitation and temperature in Yunnan in the spring is 0.312 and -0.444 respectively, both passing the 0.05 and 0.01 significance level test. When AO is situated in the negative anomaly status, the middle-latitude air pressure is low, and the air pressure in the polar area is relatively high, the meridional circulation is popular in the middle latitude, cold air is active, but the cold air is easterly and northerly in the southward path, having weak influence on atmospheric temperature in Yunnan. Therefore, when AO is in negative abnormality, the spring in Yunnan is characterized by high temperature and little rain, thus easily resulting drought [32]. Studies have discussed the impact of AO on the continuous seasonal drought in 2009-2010. In the winter of 2009/2010, AO index had the extremely low negative value, reaching the extremely small value since 1951. In such case, the west air flow in the polar vortex and medium and high latitude is weak, the meridional circulation easily appears in the medium and high latitude region, and the cold air movement is frequent. Besides, the subtropical south branch has weak west wind and the south trough intensity is reduced, which is unbeneficial for the increase of the precipitation in Yunnan.

Such circulation abnormality happens because tropical West Pacific Ocean and tropical Indian Ocean are situated in the temperature rise period from the autumn and winter of 2009 to the spring of 2010, resulting in the intensification of southwest air flow in the southeast coastal area of China, while the space above South China and Central China regions are under the control of low trough, thus it is controlled by the northwest air flow and sinking air flow behind the trough in the east of the plateau, so that the vapor from the Bay of Bengal is difficult to reach Yunnan-Guizhou Plateau, resulting in the long-term precipitation deficiency in this region [35]. On the other hand, the quasi-stationary planetary wave guide was quite strong in the polar place at 
the medium and high latitude from the winter of 2009 to the spring of 2010, while low-latitude wave guide was weak, so the latitudinal average westerly was intensified in the medium and high-layer of the convection layer near $35^{\circ} \mathrm{N}$, resulting in very large negative value of AO index, East Asia cold air is weak and has the easterly path, while the cold air movement is weak around the Yunnan-Guizhou Plateau, thus resulting in weak cold air movement in the southwest region of China and long-term precipitation deficiency and severe drought [36].

In addition to the above circulation abnormality reasons, the development period of El Nino event, attenuation period of La Nina event and relatively high sea temperature of the Indian Ocean are beneficial for the generation and development of drought in Yunnan. Therefore, the extreme drought is related to circulation abnormality, and is also influenced by external signals, such as ENSO [37].

\section{Conclusions and Discussion}

1) The climatic change characteristics in Yunnan manifest that the annual average temperature basically has the rise trend, annual precipitation is reduced in the east, northeast, south and west of Yunnan, particularly there is obvious decrease trend of the precipitation in the rainy season. Other factors related to drought, such as sunshine hours influencing the evaporation, have the trend of decrease in the north and increase in the south of Yunnan since 1961, taking Ailao Mountain as the boundary, having the increase trend in the west and south of Ailao Mountain, and decrease trend in the north and east of Ailao Mountain.

2) By using different drought indexes, the drought in Yunnan often happens in the dry season and the extreme drought event often manifests the characteristics of seasonal continuous drought. Since the 1960s, the general trend of drought in the dry season in Yunnan is humidity mitigated and drought intensity increased.

3) The impact factors of drought abnormality in Yunnan are related to the large-scale atmospheric circulation (AO, MJO etc.), abnormal sea temperature of Pacific Ocean and Indian Ocean, vapor transportation path, intensity of equatorial convection and the building of summer monsoon. In the winter and spring, the cold air in the northern hemisphere runs southward in the medium and high latitude and low-latitude warm and wet air is transported northward, which become the main factor of influencing the drought in Yunnan, summarized in Figure 3 of the climatic model.

The direct reason for the drought in a region in a time period is insufficient natural precipitation. Many meteorological scholars have analyzed the causes for drought in Yunnan by starting from the convection factors influencing the precipitation abnormality, holding that the abnormality of low-latitude and medium and high-latitude atmospheric circulation allocation has resulted in the abnormality of time-space distribution for precipitation in Yunnan Region, thus resulting in the happening of the drought events. But Yunnan is situated in low latitude, and is adjacent to Qinghai-Tibet Plateau, there is little study on the impact of the change of large-scale topographic effect of the plateau on the regional drought and on the impact of the sea temperature and circulation in the southern hemisphere, thus further elaborate study is required. In addition to the precipitation factor, the influencing mechanism of other factors influencing 


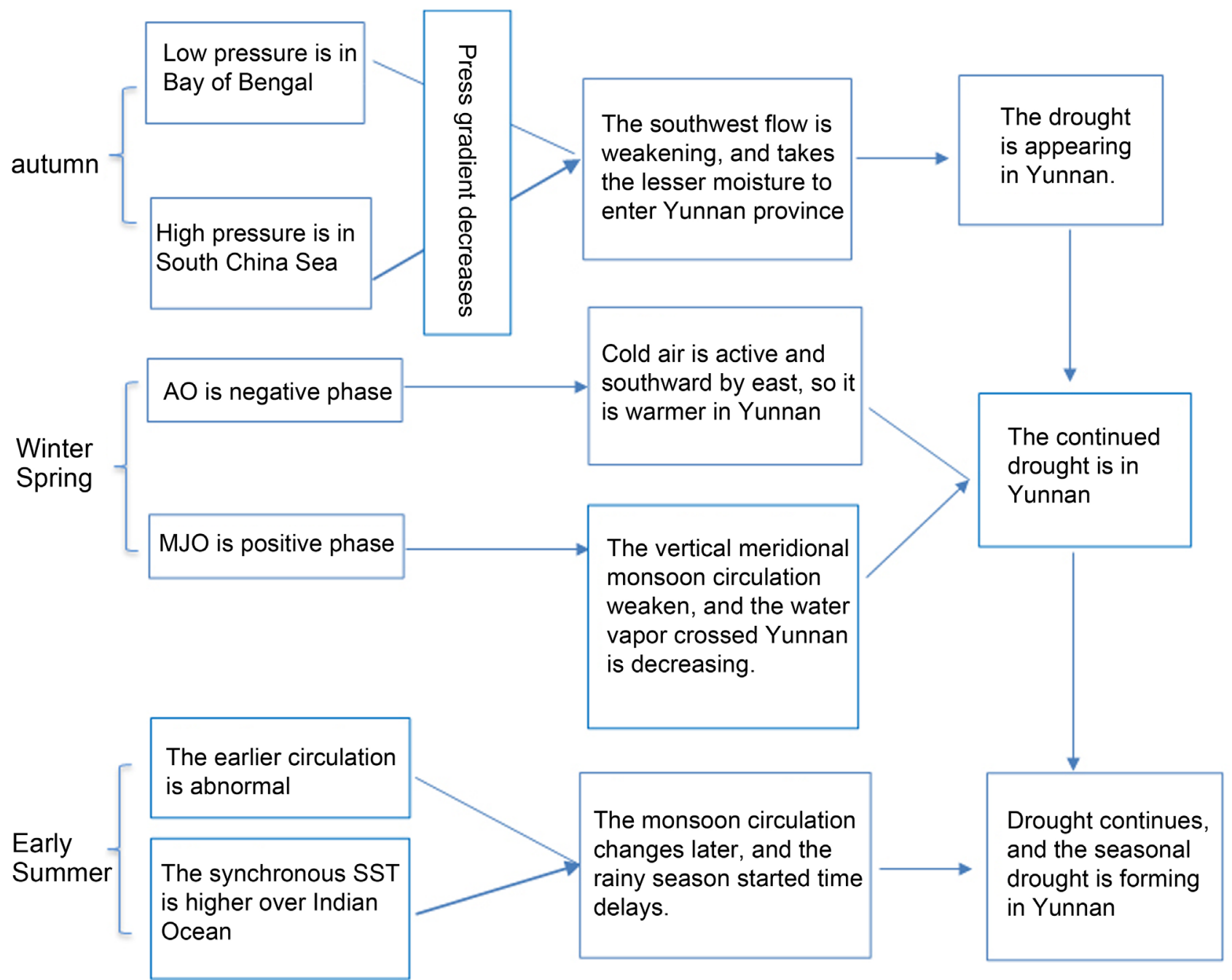

Figure 3. The concept map of Yunnan severe drought causes.

drought, such as atmospheric temperature, wind velocity, solar radiation and ground surface situations, isn't so clear.

In addition, it is required to deepen the study on the characteristics of continuous drought in Yunnan, that is, the study method for objective identification and analysis of time-space distribution characteristics, duration, happening frequency and intensity change, and the objective assessment method for the drought events in Yunnan.

\section{Fund}

NFSC (U1133603, 41365007, 41440034), Dry weather fund (IAM201402), Special industry (GYHY201306022), Key laboratory of agricultural information technology projects (201403).

\section{References}

[1] Qin, J., Ju, J.H. and Xie, M.E. (1997) The Meteorology and Climate of Low-Latitude Plateau. Meteorological Press, Beijing, 14-15.

[2] Zhang, Q., Pan, X.B. and Ma, Z.G. (2009) The Drought. Meteorological Press, Beijing, 1-72. 
[3] Zheng, J.M., Ren, J.Z. and Zhang, W.C. (2010) Analysis on Variation Characteristics of Temperature and Rainfall in Yunnan in the Last 100 Years. Journal of Catastrophology, 25, 24-31.

[4] Ding, Y.H., Ren, G.Y., Shi, G.Y., et al. (2006) National Assessment Report of Climate Change (I): Climate Change in China and Its Future Trend. Advance in Climate Change Research, 2, 1-9.

[5] Chen, Y., Duan, X., Dong, W.J., et al. (2013) Trend of Extreme Temperature under Difference Climate Backgrounds in Yunnan Province. Meteorological Sci Technol, 41, 126-130.

[6] Wu, S.H. and Zhang, Y.P. (2009) Special Environment Pattern and Ecological Distribution in Longitudinal Range-Gorge Region. Science Press, Beijing.

[7] Zhang, W.C., Zheng, J.M., Ma, T., et al. (2015) Temporal and Spatial Distribution and Variation of Extreme Temperature in Yunnan Province from 1961-2010. Resources Science, 37, 710-722.

[8] Duan, X. and Tao, Y. (2008) The Climate Change of Yunnan over the Last 50 Years. Journal of Tropical Meterology, 28, 243-250.

[9] Liu, Y., Zhao, E.X., Huang, W., et al. (2010) Characteristic Analysis of Precipitation and Temperature Trend in Yunnan Province in Recent 46 Years. Journal of Catastrophology, $25,39-44$.

[10] Tao, Y. and He, Q. (2008) The Temporal and Spatial Distribution of Precipitation over Yunnan Province and Its Response to Global Warming. Journal of Yunnan University, 30, 587-595.

[11] Liu, X., Yu, C. and Ren, G. (2007) Variation of Evaporation Capacity and Influence Factors in Hebei Province between 1963-2003. Arid Land Geography, 30, 507-512.

[12] Zhou, S., Zhang, R. and Zhang, C. (1997) Meteorology and Climate. Higher Education Press, Peking.

[13] Huang, Z. (2010) Research on Relationships between Monthly Evaporation and Conventional Meteorological Elements during Dry Season in Yunnan. Progress in Geography, 29, 138-144.

[14] Zhang, W., Zheng, J. and Ma, T. (2015) Spatial-Temporal and Decadal Change in Sunshine Resource from 1961 to 2010 in Yunnan. Resources Science, 37, 710-722.

[15] Huang, Z., Zhong, C. and Zhang, M. (2013) Applicability Analysis for Several Drought Indices to Agricultural Drought Evaluation during the Severe Drought Year in Yunnan. Chinese Journal of Agrometeorology, 34, 221-228.

[16] Ren, J., Huang, Z. and Zheng, J. (2014) Analysis on Drought Climate Change in Yunnan Based on Relative Moisture Index. Chinese Journal of Agrometeorology, 35, 567-574.

[17] Huang, Z. (2010) Dry-Wet Climate Change of the Dry Season in Yunnan during 1961-2007. Advances in Climate Change Research, 6, 113-118.

[18] Peng, G., Liu, Y. and Zhang, Y. (2009) Research on Characteristics of Drought and Climatic Trend in Yunnan Province. Journal of Catastrophology, 24, 40-44.

[19] Zhang, W., Zheng, J. and Ren, J. (2013) Climate Characteristics of Extreme Drought Events in Yunnan. Journal of Catastrophology, 28, 59-64.

[20] Tao, Y., Zheng, J., Wan, Y., et al. (2006) Evolution Feature of the Rainy Season Onset Time of Yunnan Province. Climatic and Environmental Research, 11, 229-235.

[21] Liu, Y., Zhao E., Huang, W., et al. (2007) General Circulation Characteristic before Beginning of Yunnan Rainy Season. Arid Meteorology, 25, 17-22.

[22] Liu, Y., Zhao, E., Yang, S., et al. (2007) Diagnoses of the Severe Drought over Yunnan Area in the Early Summer of 2005. Journal of Tropical Meteorology, 13, 93-96.

[23] Zheng, J., Zhu, H. and Cao, J. (2007) Research on the Relationship between the May Rain- 
fall of Yunnan Province and the Global Sea Surface Temperature. Journal of Yunnan University, 29, 160-166.

[24] Liu, Y., Zhao, E., Huang, W., et al. (2007) The Lows over the Bay of Bengal in Early Summer and Beginning Time of Rainy Season of Yunnan Province. Plateau Meteorology, 26, 572-578.

[25] Zhang, W., Zheng J., Ma, T., et al. (2014) Composite Characteristics of the Abnormal Circulation in May between Extreme Drought Years and Rainy Years of Yunnan. Plateau Meteorology, 33, 916-924.

[26] Liu, Y. (2000) The Climatic Feature Analysis of Yunnan Rainy Season Early or Late Onset. Meteorology, 26, 45-49.

[27] Zhang, W.,Wan, Y., Ren, J., et al. (2011) Analysis of Water Vapor Transportation and Its Influence on Rainfall Anomaly in Yunnan during Autumn and Winter of 2009. Plateau Meteorology, 30, 1534-1542.

[28] Ren, J., Xiao, Z., Zhang, W., et al. (2013) Relationship between the Air Column Precipitable Water in the Southwest Area and the Rainfall over Longitudinal Range-Gorge. Journal of the Meteorological Sciences, 33, 442-448.

[29] Zhang, W., Zheng, J., Wan, Y., Ren, J., Chen, Y. and Ma, T. (2014) The Distribution of Water Resources in Low-Latitude Plateau under the Background of Climate Change. Meteorology Press, Peking.

[30] Zhang, W., Ma, T., Zheng, J., et al. (2014) The Characteristics of Water Vapor Flux in Autumn and Its Relationship with Precipitation in Yunnan. Meteorological Monthly, 40, 336348.

[31] Ai, Y., Jin, S., Zheng, J., et al. (2012) Asymmetric Variation of the Circulation over the South China Sea and the Bay of Bengal and Its Impacts on the Serious Drought in Southwest China in Autumn of 2009. Journal of Tropical Mereorology, 28, 594-602.

[32] Zheng, J., Zhang, W., Wan, Y., et al. (2013) Compareative Analysis on Abnormal Circulation in Spring in Extreme Drought Year of Yunnan. Plateau Meteorology, 32, 1665-1672.

[33] Sun, G., Li, Z. and Feng, J. (2014) Relationship between Atmospheric Low-Frequency Oscillation and Two Severe Drought Events in Southwest China. Plateau Meteorology, 33, 1562-1567.

[34] Lu, J., Ju, J., Ren, J., et al. (2012) The Influence of the Madden-Julian Oscillation Activity Anomalies on Yunnan's Extreme Drought of 2009-2010. Earth Science, 42, 599-613.

[35] Ju, J., Lu, J., Xie, G., et al. (2011) Studies on the Influence of Persistent Anomalies of MJO and AO on Drought Appeared in Yunnan. Journal of Arid Meteorology, 29, 401-406.

[36] Huang, R., Liu, Y., Wang, L., et al. (2012) Analyses of the Causes of Severe Drought Occurring in Southwest China from the Fall of 2009 to the Spring of 2010. Chinese Journal of Atmospheric Science, 36, 443-457.

[37] Tao, Y., Zhang, W., Duan, C., et al. (2014) Climatic Causes of Continuous Drought over Yunnan Province from 2009-2012. Journal of Yunnan University, 36, 866-874. 
Submit or recommend next manuscript to SCIRP and we will provide best service for you:

Accepting pre-submission inquiries through Email, Facebook, LinkedIn, Twitter, etc. A wide selection of journals (inclusive of 9 subjects, more than 200 journals) Providing 24-hour high-quality service

User-friendly online submission system

Fair and swift peer-review system

Efficient typesetting and proofreading procedure

Display of the result of downloads and visits, as well as the number of cited articles Maximum dissemination of your research work

Submit your manuscript at: http://papersubmission.scirp.org/

Or contact acs@scirp.org 